\title{
ARTículo
}

\section{Fiesta Internacional de Teatro en Calles Abiertas (Fiteca): Construcción de formas económicas alternativas y configuración de identidad territorial desde el teatro}

\section{International Theater Festival in Open Streets (Fiteca): Construction of alternative economic forms and configuration of territorial identity from the theater}

\author{
Thalia Jara Mogollón \\ https://orcid.org/0000-0001-5229-0845 \\ thalia.jara@unmsm.edu.pe
}

Ela de Fátima Venturo Gómez

https://orcid.org/0000-0001-6751-395X

ela.venturo@unmsm.edu.pe

Juan Manuel Pineda Torres

juan.pineda@unmsm.edu.pe

\author{
Cinthya Mishel Chincha Rodríguez \\ cinthya.rodriquez@unmsm.edu.pe
}

Arabela Micol Villanueva Soto

https://orcid.org/0000-0003-4275-5498

arabela.villanueva@unmsm.edu.pe

Universidad Nacional Mayor de San Marcos

\section{RESUMEN}

El presente estudio tiene como objetivo responder si los procesos artístico-culturales teatrales desenvueltos, desde Fiteca, constituyen formas económicas alternativas que configuran una identidad territorial en el barrio de La Balanza, en el distrito de Comas, Lima. A efectos de este, se hace un abordaje que comprende la trayectoria o memoria histórica de la experiencia de la comunidad Fiteca, el análisis de su estructura y organización interna, las relaciones externas que teje con agentes y actores externos, así como su relación con el territorio; y, sus perspectivas a futuro. Para ello se empleó entrevistas semiestructuradas a través de las cuales se dialogó con los miembros y colaboradores de la organización con el fin de revelar las dinámicas desenvueltas dentro de Fiteca. Así, se pudo atisbar que las prácticas artístico-culturales y las dinámicas desenvueltas entorno a ellas por la comunidad Fiteca se basan en formas de trabajo cooperativo y voluntario como pilares fundamentales de producción y reproducción de sus dinámicas que, con el paso de los años, han configurado una identidad territorial en los habitantes de La Balanza. Asimismo, su organización interna tiene un basamento democrático en la medida que recoge las opiniones e inquietudes de todos sus miembros y participantes a través de asambleas generales, donde cada uno tiene voz y voto. Además, se da una división del trabajo mediante la organización de grupos de trabajo o comisiones por cada área. Finalmente, los eventos y actividades que desarrolla la comunidad están abiertos al público en general y sin costo alguno. En función a las características descritas, Fiteca comparte lógicas con las formas económicas alternativas como la economía social.

Palabras clave: FITECA; procesos cultural-artístico teatrales; identidad territorial; actores locales; formas económicas alternativas; economía social.

(C) Los autores. Este artículo es publicado por ISHRA, Revista del Instituto Seminario de Historia Rural Andina de la Facultad de Ciencias Sociales de la Universidad Nacional Mayor de San Marcos. Este es un artículo de acceso abierto, distribuido bajo los términos de la licencia Creative Commons Atribución 4.0 Internacional (CC BY 4.0) [https://creativecommons.org/licenses/by/4.0/deed.es] que permite el uso, distribución y reproducción en cualquier medio, siempre que la obra original sea debidamente citada de su fuente original. 


\begin{abstract}
The present study aims to answer if the cultural-artistic theatrical processes developed, from Fiteca, constitute alternative economic forms that configure a territorial identity in La Balanza. For the purposes of this, an approach is made that includes the trajectory or historical memory of the experience of the Fiteca community, the analysis of its structure and internal organization, the external relations that it weaves with external agents and actors, as well as its relationship with the territory; and its future prospects. For this, semi-structured interviews were used through which dialogue with the members and collaborators of the organization was used in order to reveal the dynamics developed within Fiteca. Thus, it was possible to discern that the artistic-cultural practices and the dynamics developed around them by the Fiteca community are based on forms of cooperative and voluntary work as fundamental pillars of production and reproduction of their dynamics that, over the years, They have configured a territorial identity in the inhabitants of La Balanza. Likewise, its internal organization has a democratic foundation insofar as it collects the opinions and concerns of all its members and participants through general assemblies, where each one has a voice and vote. In addition, there is a division of labor through the organization of working groups or commissions for each area. Finally, the events and activities that the community develops are open to the general public and at no cost. Based on the characteristics described, Fiteca shares logic with alternative economic forms such as the social economy. Keywords: FITECA; theatrical cultural-artistic processes; territorial identity; local actors; alternative economic forms; social economy.
\end{abstract}

\title{
Introducción
}

En un contexto donde las promesas de bienestar, desarrollo y progreso social existen como simples utopías, cada vez más lejanas, y los discursos modernizadores pierden credibilidad, emergen movimientos y acciones colectivas, con posturas críticas que plantean nuevas formas de entender la vida y, con ello, las relaciones sociales, económicas, políticas y culturales, así como una nueva forma de relacionamiento con el entorno natural.

En el Perú, en los últimos años, se ha visto una fuerte presencia de esfuerzos colectivos desde las y los mismos ciudadanos organizados, centrados principalmente en las periferias de Lima. Pinedo (2015), se refiere a este proceso como la emergencia de iniciativas de acción colectiva de carácter cultural desde la sociedad civil en la llamada ciudad popular, en contraposición con la fragmentación social que caracteriza a una urbe como Lima. Una ciudad llena de desigualdades y contrastes, donde formas de vida y ocupación diversas conviven a escasos metros de distancia, sin ninguna relación mutua. (Gutiérrez, Fontcuberta y Villar, 2016)

Asimismo, dichas iniciativas sociales responden, en gran medida, a un enfoque cultural para la construcción de una comunidad. Los estudios teatrales realizados, mencionan que, por las décadas del setenta, ochenta y noventa del siglo pasado se observó el surgimiento de diversos grupos teatrales que, en su mayoría, tenían en su origen la influencia de las actividades culturales promovidas por las parroquias de los barrios más pobres de Lima, y surgidas con las migraciones de los sesentas. (Malca, 2011)

Uno de esos grupos es La gran marcha de los muñecones, que surge por los años noventa en el distrito de Comas, con el objetivo de crear, a través del arte, un espacio de encuentro para todas y todos, superando la falta de salas de teatro y otras alternativas de entretenimiento cultural en el barrio donde se desarrolla. En 2002, crean y organizan la Fiesta Internacional de Teatro en Calles Abiertas (Fiteca), que se desarrolla en el barrio de La Balanza.

Benza (2011), indica que muchos creadores asumieron que el teatro debía ofrecer más que un mero entretenimiento y tenía que convertirse en un vehículo, a través del cual, poder visibilizar y reflexionar sobre lo que estaba pasando en la sociedad. 
El barrio de La Balanza es un fenómeno urbano singular liderado por organizaciones comunitarias locales que trabajan en pro del fortalecimiento social desde la cultura (Merladet, 2017). Precisamente ahí reside la importancia de analizar las dinámicas artísticas, principalmente teatrales, que se desarrollan en este lugar desde hace algunas décadas, siendo una de las más importantes el Fiteca. Por lo que, en la presente investigación, se tomará como caso de estudio esta experiencia.

En la revisión de la literatura sobre ella, se rescata una primera aproximación desde la arquitectura. Torres (2018), señala que hay una búsqueda de expresiones de arte «alternativo o urbano» (p. 13), estrechamente ligada al sentimiento de colectividad entre los pobladores de La Balanza, y es por esta razón que Fiteca se ha afianzado en la población, tanto que se ha convertido en un tema de identidad propia del lugar. Por su parte, Rodríguez (2020), también desde un estudio arquitectónico, se enfoca en evaluar cómo repercute esta interacción en el barrio, visto en la transformación de lo urbano y arquitectónico que impulsa el progreso con variables y dinámicas sociales que favorecen la calidad de vida de los vecinos y vecinas del barrio.

En referencia a los procesos de intervención sobre el espacio, algunas investigaciones como la realizada por Gabella (2017), señala que se ha generado un sentimiento de pertenencia y posesión en las vecinas y vecinos, a través del trabajo en común en los talleres participativos y en los domingos comunitarios. Al cual, Ruiz (2019), añade que estas prácticas devienen en la configuración de sentido de pertenencia y de lo colectivo, desarrollo y fortalecimiento de capacidades, surgimiento y consolidación de liderazgos locales.

También, en otra investigación, se indica que como referentes del uso cotidiano del barrio, se determinan la identidad y los nodos de apropiación que se dan de acuerdo al desarrollo artístico de los pobladores y la influencia de este sobre su entorno físico (Cunyas y Cárdenas, 2018). Así, estas dinámicas de apropiación y transformación del espacio, responden a otro objetivo importante de Fiteca: integrar los diferentes asentamientos humanos (hoy barrios culturales), que se encuentran fragmentados social y espacialmente; formalizar y potenciar la apropiación de los espacios públicos y consolidar un sistema urbano integral. (Rodríguez, 2015)

Otra investigación, desde las artes escénicas, señala que el Fiteca tiene como objetivo crear dinámicas que acompañen a los vecinos y vecinas en todas aquellas acciones que puedan ser creadoras de colectividad, integración, identidad y participación en el barrio. (Navarro, 2014)

Al respecto, un estudio, desde la etnografía, realizado por Bild (2012), indica que se ha observado que los niveles de cohesión social, organización y compromiso cívico se ven incrementados debido a la presencia de dicha organización en el barrio.

También, se observa la resocialización de ciertos actores. Al respecto, Casallo (2013), desde una mirada sociológica, señala que se prioriza la resocialización de individuos que antes de la existencia del surgimiento del fenómeno Fiteca estaban enajenados del sistema, como es el caso de los pandilleros. Asimismo, Pinedo (2015), refiere que Fiteca visibiliza la emergencia de nuevas alternativas basadas en el arte y a partir de las redes, la ofrenda y la autogestión, como una reivindicación de la agencia de la sociedad civil en los procesos de transformación de su propio entorno. Esta iniciativa viene transformando a La Balanza, en un barrio vivo que se opone a la «ciudad formal», donde hoy impera el miedo y el desencuentro (Cubas, 2014).

Es pertinente precisar, que el colectivo no presenta alternativas, sino la posibilidad de que las mismas personas, luego de lo que han observado, se cuestionen acerca de su propia realidad: se convierten en actores que generan su propio cambio. (Gonzales, Guerra y Valle, 2012) 
Finalmente, un aspecto importante que se destaca en los estudios realizados sobre esta experiencia, y en lo que nos interesa examinar en la presente investigación, está referido a las formas de organización y gestión de recursos que permite la reproducción de estas prácticas artístico-culturales. Sobre ello, Fioralisso (2016), refiere que la comunidad Fiteca está enmarcada en una tradición barrial de organización vecinal del distrito de Comas: la autogestión y trabajo voluntario (faena) e invitación de terceros. Así, la base del sostenimiento de esta experiencia es su vasto capital social que les permite acogerse a grandes redes de colaboración afianzadas con el tiempo (Pinedo, 2015). El reto más grande que tiene este colectivo es «hacer del arte una alternativa distinta de desarrollo social, económico y humano; sin embargo, este cambio no se dará si los pobladores no se unen como comunidad, discuten sobre la problemática que los afecta y plantean soluciones de cambio». (Gonzales, Guerra y Valle, 2012, p. 27)

A partir de las investigaciones hechas sobre Fiteca, desde diversas disciplinas, se resaltan algunas conclusiones que permiten comprender las dinámicas desenvueltas desde esta experiencia en el barrio de La Balanza. Pinedo (2015), plantea que Fiteca visibiliza formas de trabajo que velan por la autonomía local y la interdependencia funcional entre actores sin constituirse como un sistema hermético; dado que las lógicas de trabajo que están detrás de ella se basan en la ofrenda que distan de las relaciones mercantiles que caracterizan las industrias culturales.

También, otra consideración relevante se refiere a que:

es necesario pensar las intervenciones de agrupaciones culturales comunitarias, como la comunidad Fiteca, como políticas culturales específicas impulsadas desde la sociedad civil más allá de la mera organización y orientación de lo simbólico; dado que, dentro de un contexto social específico, pueden ser generadoras de movilizaciones simbólicas que acaban por tener repercusiones en campos que exceden lo cultural y alcanzan los ámbitos políticos, económicos y sociales y de, esta manera, pueden llegar a constituir distintas realidades en su contexto concreto de intervención. (Fioralisso, 2016, p.14)

Si bien, se ha estudiado ampliamente las dinámicas que desenvuelve Fiteca y desde diversas perspectivas, la mayoría son abordajes arquitectónicos, artísticos y sociales con un enfoque que destaca más lo cultural. Así, en las indagaciones hasta ahora realizadas no ha sido abordado el aspecto de las relaciones económico sociales; y dentro de ello, las formas de organización y dinámicas basadas en la cooperación y colaboración desinteresada de sus miembros que nos lleva a observar características que están fuera de las lógicas de la economía de mercado, por lo que, estarían más vinculado a las lógicas que comprende a las economías alternativas ${ }^{1}$.

Para entender estas lógicas alternativas a la acumulación capitalista es necesario comprender las propuestas que nos traen los debates sobre una Economía Social y sus diferentes corrientes, variantes o vertientes teóricas. Al respecto, Coraggio nos plantea que la economía social es una noción que pretende superar la opción entre el mercado capitalista y un Estado central planificador y regulador de la economía, la cual se lograría superando la separación entre economía y sociedad.

De ese modo, esta concepción ve la posibilidad de desarrollar una socioeconomía, donde los agentes no son escindidos de sus identidades sociales, de su historia ni de su cultura. Ve la economía como inseparable de la cultura. A partir de ello, la ve como un espacio de acción

1 Al respecto, Quijano (2014). sostiene que: «Tomando en cuenta la heterogeneidad histórica y estructural del mundo, las economías alternativas surgen desde la reciprocidad en las condiciones y con los instrumentos producidos en el capitalismo. Donde, lo alternativo hace referencia a modos o sistemas de producción alternativa en relación con la dinámica del capital; y la reciprocidad, como el intercambio de fuerza de trabajo y de trabajo sin la intermediación del mercado». (p. 219) 
constituido por individuos, familias, comunidades y colectivos de tipos diversos que se mueven dentro de instituciones decantadas por la práctica o acordadas como arreglos voluntarios, que actúan que actúan haciendo transacciones entre la utilidad material y los valores de solidaridad ${ }^{2}$ y cooperación, limitando la competencia. (Coraggio, 2011)

Asimismo, Coraggio sostiene que esta economía es social porque produce sociedad y no sólo utilidades económicas, es decir, produce valores de uso para satisfacer la necesidad de los mismos productores o de sus comunidades y no está guiado por la ganancia y la acumulación de capital sin límites. Además, su gobierno interno se basa en la deliberación de miembros que cada uno/a tiene un voto, pero admite la división del trabajo y los sistemas de representación y el control de las responsabilidades. De modo que, se establece la democracia participativa como una característica esencial.

Otro aspecto importante de las organizaciones de la economía social es que desde el inicio se autodefinen como sin fines de lucro, la cual no las convierte en anticapitalistas, pero si nocapitalistas (Corragio, 2011). Estas organizaciones pueden ser de diversos tipos, dentro de la cual se encuentran las asociaciones culturales de encuentro comunitario y afirmación de las identidades como podría ser el caso de Fiteca.

Asimismo, se mencionó que la economía social tiene diversas corrientes y sus variantes, diversas matrices culturales. Dentro de la cual podemos encontrar a la economía solidaria y la economía popular como las vertientes mayores del debate latinoamericano en torno a la cuestión de las formas alternativas de producción.

Quijano establece una diferenciación entre ambas: por un lado, la economía solidaria tiene como institución central a la cooperativa, donde las reglas del mercado y del salario están más presentes, y la reciprocidad ${ }^{3}$ opera como desde fuera de las relaciones de trabajo, además se ubican política e ideológicamente en contraposición al capitalismo; y por el otro lado, la economía popular hace referencia a instituciones heterogéneas de organización de la producción, de la distribución y la relación con el mercado, donde su elemento común es que son unidas constituidas por gente que tiene relaciones primaria entre sí que forman agrupaciones pequeñas y tienden a organizarse mediante una lógica comunitaria.

Así, «lo que caracteriza a la economía popular es que las relaciones de trabajo y de distribución de recursos y del producto se organizan, en lo fundamental, en torno a la reciprocidad y vida social, las prácticas sociales cotidianas y la comunidad». (Quijano, 2011, p. 380)

En la misma línea, Germaná nos hace referencia a una economía de la reciprocidad, donde esta «constituye un vasto conjunto de organizaciones económicas que no se estructuran según la lógica de la ganancia y de la acumulación sino en función de la satisfacción de las necesidades colectivas fundamentales» (Germaná, 2015, p.179). Desde esa perspectiva, nos dice que se funda en la lógica de la reciprocidad o comunidad, donde el eje principal de este tipo de organización

\footnotetext{
2 La solidaridad se entiende como «relación material, y no solo intersubjetiva, que vincula a los individuos que forman parte de un grupo, una asociación o una comunidad y los lleva a cooperar entre ellos para lograr fines comunes». (Germaná, 2015, p.175)

3 La reciprocidad es entendida como un tipo particular de relaciones sociales de producción que se diferencia de conceptos como la pobreza, marginalidad e informalidad, en la medida que no entra en las lógicas de la acumulación capitalista, pero tampoco es un eufemismo para la pobreza; ya que, esta responde al problema de la distribución; la informalidad es una relación social capitalista de producción que no es reconocida legalmente como tal; y la marginalidad se vincula con una forma específica que asume la fuerza de trabajo que ha sido desplazada de la producción por la lógica de acumulación capitalista. Por ende, «la reciprocidad es un tipo especial de intercambio fundado en el valor de uso de los bienes, en ese sentido desaparece el papel del mercado como eje del intercambio de bienes según su valor de cambio». (Germaná, 2015,178)
} 
económica es la comunidad. Así, la que prima es la solidaridad, «la lógica de la cooperación y no la del beneficio personal». (p.176)

De ese modo, estas propuestas teóricas que plantean lógicas económicas alternativas a la racionalidad económica capitalista nos permiten entender las relaciones económicas sociales desde una perspectiva diferente y alternativa, y nos dota de nuevos elementos conceptuales y categorías para analizar estas relaciones.

A partir de lo mencionado y en función a las investigaciones revisadas, el presente trabajo de investigación pretende responder a la siguiente interrogante: ¿Qué procesos cultural-artísticos teatrales desenvueltos, desde Fiteca, constituyen formas económicas alternativas que configuran una identidad territorial en La Balanza?

La metodología empleada para la presente investigación es de corte exploratorio, transversal y descriptivo con énfasis en el método cualitativo, donde se va indagar en las relaciones internas y externas desenvueltas desde esta experiencia. Asimismo, visibilizar la configuración de una identidad territorial, así como la posibilidad de construcción de formas económicas alternativas en el marco de una sociedad consumista regida por una economía de mercado en constante expansión.

Las técnicas que se usaron, durante la pesquisa, fueron entrevistas semiestructuradas, a través de las cuales se dialogó con los miembros fundadores de Fiteca y algunos miembros colaboradores; así como la revisión de información virtual presentada por la organización y documentos internos de ella, junto al arqueo de literatura dedicada a su experiencia.

La estructura de la investigación se divide en las siguientes partes: primero, se presenta la trayectoria de Fiteca desde sus inicios, el contexto y su evolución a lo largo de los años hasta el día de hoy; segundo, se indaga sobre la estructura y la organización interna de la experiencia; tercero, se analiza sus relaciones con actores y agentes externos y su relación con el territorio donde desarrolla sus dinámicas; y cuarto, se indaga sobre sus objetivos a corto y largo plazo. Finalmente, se presentan las conclusiones del estudio.

\section{Memoria histórica}

El cerro La Balanza, uno de los espacios que dio inició a la historia del distrito de Comas hace 61 años, lugar donde existen 16 asentamientos humanos, desde el 2002 hasta la actualidad, se ha convertido en el corazón del Fiteca. El parque Tahuantinsuyo, ubicado en la cuadra 26 de la avenida Puno, año tras año en el mes de mayo se vuelve el foco del considerado, hoy en día, como el festival de teatro callejero más grande de América Latina. Un lugar donde antes dominaba el pandillaje ahora lo domina la cultura, denotada con la existencia de un busto de César Vallejo en su paisaje. Dicho proceso de resocialización de los denominados pandilleros no ha pasado desapercibido, por ello, Casallo (2013), analiza este suceso:

Un desarrollo admirable de esta experiencia ha sido la relación con las pandillas del distrito. Al inicio, los vecinos negocian con los pandilleros para que no perturben el festival; posteriormente algunos se han incorporado como parte de la seguridad. Ahí donde las políticas públicas de seguridad, los colegios y las familias aisladas han fallado, quienes movilizan el Fiteca han logrado consolidar. (Casallo, 2013, p. 105)

Fiteca lleva 19 años desarrollándose, impartiendo arte y cultura en el barrio de La Balanza, por ello, es relevante destacar un antecedente crucial para la existencia de este magno evento, la 
creación del centro cultural La Gran Marcha de los Muñecones, en 1990, espacio que nace como grupo de teatro urbano, que se sitúa precisamente en La Balanza. Siendo uno de sus fundadores, por ese entonces, Jorge Rodríguez, quien en la actualidad con la dirección de Patricia Beltrán son los principales rostros de impulso del Fiteca.

Es relevante aquí hacer mención que la idea de la fundación de un espacio teatral urbano, es una propuesta de teatro popular periférico en contraposición a una lógica del teatro entendido como un espacio de acceso solo para las elites, un lujo, un evento que solo pudieran entender los cultos. Estas particularidades y objetivos de trabajo del teatro popular, justamente, son base del análisis de la tesis de Malca (2008), quién plantea algunas características para la identificación de estos espacios, los cuales son:

La capacidad de plantear discursos que pueden transmitir referentes de la identidad social-urbana marginal. La cual surge a partir de los siguientes elementos: A) Un marco de vivencias comunes a grupos sociales. B) Percepción y consciencia de la realidad en la que viven. C) Marco culturales de procedencia (teniendo en cuenta que se trata de poblaciones que han surgido mayormente como producto de la migración del campo a la ciudad). (Malca, 2011, p. 179)

Pero también es importante esta mención por el periodo donde se desarrolla la fundación de "La Gran Marcha de los Muñecones", pues en 1990 fue el año del inicio del gobierno de Fujimori, época a su vez del conflicto interno con Sendero Luminoso. Por ello, este tipo de manifestaciones culturales pasaron a ser senderizadas, y la creación del festival Fiteca recién pudo concretarse en el 2002, es decir, posterior a la finalización del gobierno fujimorista.

Jorge Rodríguez, al respecto indica: «existía miedo de los vecinos cuando les comentábamos sobre nuestros proyectos culturales, durante el gobierno de Fujimori teníamos que reunirnos de manera clandestina por miedo a que nos acusen de conspiración contra el gobierno o presunción de terrorismo, ello incluso tras la caída del régimen fujimorista aún estaba interiorizado en los vecinos y vecinas, lo cual fue nuestro primer y principal reto para construir FITECA». ${ }^{4}$

Por lo mencionado Fiteca nace con el objetivo principal de la reinvención de la memoria del barrio y así lograr la construcción del barrio cultural. Durante estos 19 años, el trabajo ha sido arduo con los vecinos y vecinas de La Balanza:

Hoy en día durante la realización del festival los vecinos conviven con los artistas, asisten y participan en los distintos talleres, conversatorios y presentaciones de las agrupaciones culturales e incluso de las reuniones para preparación del evento que se hace comúnmente con 6 meses de anticipación. ${ }^{5}$

Si bien, Fiteca nace en el 2002, con los esfuerzos de sus impulsores en conjunto con los vecinos y vecinas de La Balanza; pero es recién en su edición del 2007, bajo el slogan «Una vez más Arte entre cerros», que inicia un apoyo institucional de parte de la Municipalidad Distrital de Comas, y que hasta la actualidad se mantiene. Su edición del 2008, denominada: «Donde los cerros murmuran colores», inicia la fundación de colectivos de los mismos miembros activos en la construcción de Fiteca, es decir, las personas que de manera independiente se juntaban para el impulso de este proyecto comenzaron a asociarse en función de sus tareas, naciendo en ese entonces el Colectivo CITIO, conformado por estudiantes de arquitectura de la UNI. Asimismo, en las ediciones del 2009, «Un sueño que nos hace familia, un barrio que se hace poema»; y la del

4 Entrevista a Jorge Rodríguez el 14 de agosto de 2020.

5 Entrevista a Jorge Rodríguez el 14 de agosto de 2020. 
2010, «Mientras más profunda nuestra memoria, más grandes nuestras utopías y más brillante nuestro presente»; podemos observar en los slogans el énfasis en los objetivos de búsqueda por sus impulsores, la reinvención de la memoria y la construcción del barrio cultural, desaprender para aprender.

En la edición del 2011, «Juntemos nuestras experiencias, que nos impulse el amor, construyamos imaginarios», el festival se extiende por primera vez de una semana a 10 días. Sin embargo, en la edición del 2012, «Recogiendo experiencias, anécdotas, aciertos y desaciertos para volver a impulsarnos unos 10 años más», el evento de un magno festival pasó a ser un conversatorio de reflexión de 3 días desarrollado en La Balanza, en la primera semana de mayo, haciendo denotar una leve caída del espacio; pero ello, sería tan solo un traspié que se retoma con mayor fuerza en la edición del 2013: «Vamos todos por el barrio cultural»; y es en su edición del 2014, «13 años construyendo barrio», donde el festival pasaría a ser presentado en 2 sedes distintas dentro del mismo distrito de Comas, siendo la segunda sede el barrio de El Carmen, en el complejo deportivo José Carlos Mariátegui, en el kilómetro 13 de la avenida Túpac Amaru.

Este esquema se volvió a repetir durante las ediciones del 2015, «Nuestros padres migrantes sembraron barrios en los cerros. Hoy, al abrir el alma habitan cantos, ritmos y sabidurías»; y 2016, «El sembrado: 15 años sembrando la semilla de la cultura en los barrios», las cuales tienen una particularidad en función del objetivo de los impulsores. Jorge Rodríguez, en una entrevista realizada por un medio local durante la realización de la edición del 2015 anuncia que, de cara a la decimoquinta edición, comenzará a expandir el proyecto a otros distritos en miras de la construcción de más barrios culturales. Es así que recién en el 2017, «Haciendo del barrio como un latido», se inicia la descentralización del evento, incluyendo ahora a los distritos de La Molina y Pucusana, ambos eventos realizados con el apoyo de sus respectivas municipalidades.

A partir de la edición del 2018, «Construyamos los cerros mágicos», el evento se traslada a otras regiones del Perú, siendo en el 2018, la región Ica la primera en sumarse con 3 sedes (Chincha, Pisco e Ica); y en la edición del 2019, «Desde las raíces al encuentro del cóndor con el águila», se suma la región Cusco. Asimismo, la edición del 2020 no ha podido organizarse, por efectos de la pandemia provocada por el COVID-19; pero existe una proyección de los distintos grupos artísticos, a través de las redes sociales de FITECA, pero de manera esporádica no en la lógica de un festival itinerante.

Como se observa, a lo largo de todo este recorrido histórico de Fiteca se ha logrado acrecentar las redes de apoyo para garantizar el desarrollo del mismo año tras año, es correcto usar el término de redes de apoyo pues Fiteca no tiene fines de lucro y si bien dentro del desenlace del festival existen espacios de actividades económicas, los que asumen estos espacios son los vecinos y vecinas de La Balanza, los ingresos que generan son directos ingresos para los mismos vecinos no para la organización de Fiteca. En la entrevista realizada a Jorge Rodríguez nos hizo mención que existe una persona del barrio de La Balanza quién se encarga de coordinar con los vecinos y vecinas de consensuar quienes necesitan generar actividades económicas (que son principalmente venta de alimentos) durante la realización del festival para planificar la distribución de los espacios, por ende, no existen alquileres de los mismos. Lo mencionado demarca una red de solidaridad que escapa claramente de una lógica de mercado, porque es común ver en festivales culturales o musicales cómo se alquilan espacios a distintas marcas y estos sobregiran precios para generar ingresos de manera 
desproporcionada durante su realización. Este pequeño ejemplo también nos demuestra que la estructura más básica y esencial para que exista Fiteca es la comunidad de La Balanza, incluso hoy en día no es desatinado decir que dicha comunidad ha interiorizado a Fiteca como parte de su identidad, y por ende ello hace que asuman de manera colectiva la responsabilidad para lograr su realización.

Para llegar a este punto hay que partir diciendo que es un resultado gracias a la reflexión crítica, y resultado de ello, la acción; que ha generado el teatro popular en ellos durante todo este tiempo, porque como hemos anotado ya existía un colectivo de teatro popular una década antes de la existencia de Fiteca en el 2002, así que no podríamos decir que ha sido un proceso rápido más aún por el contexto histórico ya anotado, porque la red de apoyo de vecinos del inicio de Fiteca no es para nada símil a la actual existente, este proceso deconstructivo y la vez constructivo de mirada de tu entorno, de buscar salida a las problemáticas a través del trabajo de espacios en común es gracias a la apertura que generó el arte impulsado por el colectivo de la "Marcha de los Muñecones".

Porque la realización de Fiteca es un proceso nada sencillo a pesar de la existencia actual de asociaciones de apoyo macros que serían las municipalidades o gobiernos regionales, estás asociaciones incluso no serían nada si los vecinos y las vecinas de la balanza no generan durante el año actividades de autogestión para prepararse para recibir a los artistas extranjeros, darles comida, espacios donde descansar, para el recibimiento de toda comunidad externa a su barrio. Entonces no estamos hablando de una asociación de personas que programan un evento para generar rentabilidad de su inversión, personas que quieren posicionar un nombre como marca, que quieren ofrecer cultura como producto para el consumo de los ciudadanos que tenga los recursos para adquirirlos; estamos hablando del interés individual de los distintos vecinos y vecinas que logra que se suman a las comisiones y actividades, y dichas acciones se vuelven una satisfacción colectiva que hace que refuerce su identidad como comunidad a través de este logro año tras año.

\section{Relaciones internas}

Fiteca se realiza bajo la iniciativa de agrupaciones artísticas, culturales, sociales y educativas con la finalidad de promover el arte y la cultura desde la comunidad. La Gran Marcha de los Muñecones, liderada por Jorge Rodríguez, es el grupo de soporte que busca activar barrios por medio del arte y la cultura, sin olvidar a las y los vecinos, que son parte de un movimiento comunitario artístico-cultural sin precedentes en el Perú ${ }^{6}$. Las actividades desenvueltas se basan en relaciones de reciprocidad a través del arte y la cultura, entre las y los vecinos, artistas, jóvenes, niños y niñas.

Asumiendo un respeto al territorio y la memoria de sus fundadores, cabe resaltar la tradición y arraigo cultural de la comunidad, por ser migrantes de diversos departamentos del Perú; esto converge para la formación de un nuevo espacio e imaginario en el barrio de La Balanza. Además, se busca generar relaciones igualitarias entre los participantes de la comunidad, que integre diversidades de etnia, clase social, educación y género. Reflejándose en el desarrollo de formación artístico-cultural sin exclusión entre sus participantes.

\subsection{Aspecto económico}

En los primeros años del proyecto, el sustento económico se dio de manera autogestionaria mediante la venta de souvenirs como llaveros, posters, polos, lapiceros u otros productos, así

6 Entrevista a Mónica Miros, el 13 de agosto de 2020. 
como de comida, bingos o rifas. A su vez mediante pasacalles en mercados cercanos, donde les otorgaban dinero y alimentos.

En la actualidad, estas actividades aún se realizan durante el desarrollo del festival, contando, además, con la participación de las y los vecinos. Del mismo modo, ellos y ellas ambientan un espacio de Feria de Comidas que les da oportunidad de generar ingresos cerca de casa.

Por otro lado, cuando fue tomando fuerza, la Municipalidad de Comas registró la incidencia cultural proporcionando donaciones económicas y logísticas. Además, con la creación del Ministerio de Cultura, en el año 2010, este convoca concursos de financiamiento de proyectos culturales y Fiteca fue acreedor del apoyo durante años consecutivos.

\subsection{Aspecto institucional}

Actualmente, mantiene una estrecha relación con su comunidad, buscando realizar con artistas y vecinos el sueño del barrio cultural (Villarán, 2012). Esto es posible mediante la acción colectiva. Para el desarrollo del evento, con seis meses de anticipación al mes de mayo del año siguiente, se organizan comisiones en diversas áreas. Entre ellas, encontramos la comisión de audiovisuales, murales, económica, hospedaje, tramoya, programación de funciones artísticas, escenario, comida, transporte, dirección, entre otras.

Producto de vínculos de cooperación se institucionalizan en espacios artístico-culturales especializados en su área como, por ejemplo: Somos Minka Audiovisual el cual surge de la comisión audiovisual que viene produciendo la película El Zorzalito, cine comunitario de niños y niñas. Además, la comisión escenario formaliza el proyecto: Fitekantropus, con fines de una mejor arquitectura barrial para las y los vecinos. Por último, la comisión mural, a cargo de Mónica Miros, impulsa el festival de mujeres: Nosotras Estamos en la Calle que se desarrolla la primera semana de marzo por el día de la mujer. En estos espacios se observa la activa participación de mujeres y diversidades para su apropiación y transformación.

\subsection{Aspecto generacional}

Según Torres (2018), si bien, en estos asentamientos, no se contaba con mayores recursos económicos, esto no fue mayor obstáculo para emprender uno de los movimientos artísticoculturales más importantes de Lima en la actualidad. Por ello, desde la práctica, se busca reivindicar la memoria donde confluyen identidades centradas en la herencia cultural de las generaciones de «niños de 0 a 90 años» de La Balanza.

Asimismo, durante todo el año, las comisiones participan activamente en el mejoramiento del territorio junto a las vecinas y vecinos con la finalidad de un bienestar comunitario 7 . Producto de ello, renovaron los espacios públicos, como el Comedor Popular Virgen del Carmen, donde un grupo de mujeres cocinan diariamente el cual se configura como una alternativa por y para las familias del barrio, llegando a incidir en el desarrollo de generaciones. De manera análoga, el Complejo Deportivo José Carlos Mariátegui, sede principal de Fiteca, es protagonista de constantes cambios.

Además, crearon La Biblioteca Fitekantropus, espacio comunitario cuyo objetivo es incentivar de forma lúdica la educación y la lectura como posibilidad de apropiación del espacio de artistas e integrantes de la comunidad, especialmente de niños y niñas, en una búsqueda de integración con las próximas generaciones.

7 Entrevista a R. Amancio el 16 de agosto de 2020 


\subsection{Relaciones comunitarias}

Desde hace cinco años, se busca descentralizar las actividades artístico-culturales de Fiteca con fines de llegar a más barrios, incentivando prácticas comunitarias desde las disciplinas artísticas. Se ha llevado a cabo en los distritos de Collique y Pucusana, así como en las provincias de Ica, Ayacucho y Cusco. A partir de ello, se observa la influencia del proyecto en diversas iniciativas culturales de Lima, entre ellas la Agrupación Kilombo Artes Escénicas, en Villa el Salvador.

Organizaciones artístico-culturales de nivel nacional e internacional colaboran con una variedad de funciones como teatro, música, malabares, murales, batucada, hip hop, entre otras; generando un intercambio cultural y aprendizajes dentro del territorio. Para ello, a la llegada de artistas, la comunidad se organiza para el recibimiento y alojamiento a través de las comisiones encargadas, donde vecinos y vecinas brindan sus hogares como espacio de estadía. Una actividad producto de estas relaciones de solidaridad, es el intercambio culinario, mediante platos típicos de los departamentos de procedencia de las y los vecinos de la Balanza y $\operatorname{artistas}^{8}$. Todas estas labores se basan en vínculos de colaboración desinteresada (sin fines lucrativos) que, mediante el trabajo cooperativo y voluntario, tanto de los/as artistas y los/as vecinos/as del barrio, sacan adelante proyectos importantes como es el festival.

\section{Relaciones con actores y agentes externos, así como con el territorio}

\subsection{Relación con entidades privadas}

A lo largo de su trayectoria, Fiteca ha ido tejiendo redes de colaboración y participación tanto con los vecinos de La Balanza y los artistas interesados como con las entidades privadas, ya sean organizaciones con fines de lucro o sin fines de lucro. Sin embargo, si bien, las entidades privadas han apoyado en algunas ocasiones, estas no han sido suficientes ni determinantes; ya que, la principal fuente de sustento de Fiteca se sostiene en la autogestión y el trabajo voluntario de sus miembros, artistas y vecinos del barrio de la Balanza, quienes participan activamente y de manera desinteresada. Este aspecto del trabajo solidario también ha sido analiza por Pinedo, quien sostiene lo siguiente:

El trabajo invertido para la realización de cada actividad es dado a manera de ofrenda por los participantes del festival; es decir, una inversión voluntaria de tiempo y trabajo la cual se ha convertido en una suerte de institución en cada uno de los que participan. (Pinedo, 2015, p.36)

En ese sentido, las lógicas de trabajo que desenvuelve Fiteca se basa principalmente en la cooperación y colaboración de todos y todas que comparten el gusto por el arte y la cultura y se identifican con los ideales que persigue Fiteca de construir comunidad, intercambio y aprendizaje colectivo. De ahí que, Pinedo define a Fiteca como:

...un movimiento cultural de carácter contencioso en tanto resulta de una acción colectiva ante los sentidos de individuación. Lo que busca es romper con ellos y generar una alternativa de redefiniciones culturales ligadas al sentido colectivo y de cooperación. (Pinedo, 2015, p. 41)

Asimismo, Fiteca es un espacio abierto para todos y todas que tengan interés en buscar una alternativa de bienestar y valore el quehacer artístico y la cultura como una herramienta para tejer el cambio. Pues, en palabras de Jorge, "la cultura es vida, es elemento de reivindicación,

8 Entrevista a Richard Amancio el 16 de agosto de 2020. 
por ello, se debe buscar la dinamización cultural con el fin de reivindicar la humanidad"9. Por tal razón, las actividades artísticas que desarrollan no tienen costo alguno y están abiertas al público en general, con lo cual dista del arte mercantilizado que produce y reproduce la industria artística y cultural. Las prácticas artísticas de libre acceso y sin coste, que promueve Fiteca, también es analizada por Gutiérrez, Fontcuberta y Villar (2016), quienes refieren que:

La Fiesta Internacional de Teatro en Calles Abiertas se celebra en el Parque del Tahuantinsuyo y sin coste alguno para sus participantes, con objeto de incentivar el acceso universal al arte. (Gutiérrez, Fontcuberta y Villar, 2016, p. 113)

Si bien, la principal forma de movilizar recursos de Fiteca es a través de la autogestión y la cooperación solidaria de sus miembros, vecinos y artistas estas dinámicas no se encuentran al margen de las lógicas del mercado, sino que se desarrollan en paralelo o junto a ellas. Al respecto Pinedo (2015), desde una perspectiva sociológica, menciona lo siguiente:

la producción artística que se lleva a cabo en Fiteca no es producida para el beneficio rentable del artista a pesar de que su trabajo tenga un alto valor monetario en circuitos regulares [...]sin embargo, no estamos ante formas de trabajo que se encuentran al margen del mercado sino más bien que conviven con él. Inexorablemente, FITECA necesita también de recursos materiales, los cuales son obtenidos a partir de donaciones diversas (Pinedo, 2015, p.37)

De ahí la importancia de la cultura, para Fiteca, como un elemento fundamental en la dimensión de la vida que va permitir forjar una sociedad más justa e integrada, con individuos más conscientes de sí mismos, donde se pondere valores como el trabajo colectivo y la cooperación para alcanzar un objetivo común para todos y todas. Así, la vida cobra valor en sí mismo y como fin fundamental, donde lo económico si bien es importante no debe ser el aspecto determinante de la vida. Sobre ello, Jorge menciona que «no se debe reducir la vida a lo económico. La dimensión de la vida y la alegría va mucho más allá de la económica. Lo económico es solo es un medio» ${ }^{10}$. Para ello, el arte como el teatro, es una herramienta que lleva al individuo a cuestionarse a sí mismo y a su entorno con el fin de reflexionar y tomar conciencia de su realidad.

\subsection{Relaciones con el Estado}

Las relaciones que mantiene Fiteca con las entidades del estado han sido conflictivas, tardías e inconstantes; ya que, en un principio, la Municipalidad Distrital de Comas se constituyó en una especie de enemigo que, en lugar de brindar apoyo a los proyectos que venía promoviendo Fiteca, los veía como un rival por la aceptación creciente y la cercanía que iban generando en la población; ya que, esto le quitaba legitimidad y mérito frente a la ciudadanía. Dicha situación provocó que la municipalidad obstaculice los trámites y permisos para desarrollar las actividades; sin embargo, cuando la comunidad Fiteca se fue consolidando y su presencia cobraba fuerza en el barrio de La Balanza, la municipalidad no tardó en sumarse mediante la facilitación de permisos y donaciones de recursos o cubriendo algunos gatos de los eventos que se realizaba, por ejemplo, los gastos de comida para los artistas, los cuales muchas veces han sido conflictivas por el uso político del festival por parte de la institución. Sin embargo, este apoyo no fue significativo ni constante; dado que, los cambios en la gestión municipal traen consigo nuevas autoridades, quienes no siempre están dispuestos a apoyar o colaborar con las actividades y eventos que se viene realizando como

9 Entrevista a Jorge Rodríguez el 14 de agosto de 2020.

10 Entrevista a Jorge Rodríguez el 14 de agosto de 2020. 
es el caso de la Municipalidad Metropolitana de Lima, donde la gestión de la alcaldesa Susana Villarán promovió y apoyó algunas propuestas artísticas como la muralización, con lo cual Fiteca también se vio beneficiado en algún momento; sin embargo, al finalizar su gestión llegó una nueva autoridad, quién no mostró predisposición de apoyar dichas propuestas, así lo señalan alguno de los miembros de la comunidad Fiteca, entrevistados en el marco del trabajo de campo del presente estudio.

Asimismo, a nivel del gobierno central, los organizadores mencionan que, no ha existido predisposición ni compromiso de las autoridades competentes en apoyar, fortalecer y promover las iniciativas artístico-culturales que ha venido desarrollando Fiteca hace más de 15 años, donde el Estado brilló por su ausencia, sobre todo, en los inicios de la trayectoria de la comunidad; y recién, en los últimos años, Fiteca viene participando de algunos proyectos y concursos que promueve el Ministerio de Cultura, lo cual ha permitido cubrir solo una fracción ínfima de los gastos logísticos, siendo este un aproximado del $10 \%$ de los gastos totales, el resto es cubierto por Fiteca a través del trabajo solidario y colectivo de sus miembros, los vecinos de La Balanza y los artistas, así como actividades de autogestión y donaciones de algunas entidades como empresas de pintura, Telefónica u organizaciones sin fines de lucro como las ONG. Sobre ello, Pinedo en sus estudios sobre Fiteca menciona que:

Fiteca visibiliza formas de trabajo que velan por la autonomía local y la interdependencia funcional entre actores sin constituirse como un sistema hermético; más bien, también necesitan de los agentes externos ligados al mercado para solucionar sobre todo temas logísticos. (Pinedo, 2015, p. 38)

Finalmente, la poca presencia del Estado y sus instituciones de representación ha hecho que Fiteca se acoja y reafirme las redes de cooperación y el trabajo voluntario, que es una forma de organización vecinal presente en el barrio, para desplegar sus dinámicas. Sobre este punto Pinedo refiere que:

este divorcio entre el Estado (la Municipalidad de Comas, de Lima, o el Ministerio de Cultura) y la sociedad civil (Comunidad FITECA) se da también como una estrategia para reafirmar la cooperación de la comunidad pues, de acuerdo a su experiencia, estas redes se movilizan proporcionalmente a la inversa al grado de ayuda externa recibida en cuanto a recursos del festival. (Pinedo, 2015, p. 39)

\subsection{Relaciones con las instituciones educativas}

Es importante, también, destacar las relaciones de Fiteca con las instituciones educativas del lugar, donde desenvuelven sus dinámicas. Los miembros de la comunidad mencionan que Fiteca también trabaja con las instituciones a nivel inicial, primario y secundario, a quienes involucra en diversas actividades artísticas como los pasacalles o los talleres que principalmente se hacen en verano. Al respecto, Richard Amancio nos comenta que en estos talleres participan niños y niñas, jóvenes y adultos, pues a veces "nosotros vamos al colegio o ellos también vienen a participar de las actividades". ${ }^{11}$ Con lo cual la actividad está abierta para todos y todas que deseen ser parte de la experiencia artística y cultural de aprendizaje, reflexión e intercambio que promueve Fiteca desde hace muchos años. Así, Fiteca no sólo teje redes de colaboración y participación con los vecinos de La Balanza, sino también con las entidades y organizaciones del lugar como es el caso de las instituciones educativas. De ahí que Fioralisso menciona que:

11 Entrevista a Richard Amancio el 16 de agosto de 2020. 
La comunidad Fiteca se define a sí misma como un espacio abierto donde artistas, profesionales, académicos y vecinos se juntan voluntariamente bajo el ideal compartido de la cultura como valor fundamental para el desarrollo del barrio. (Fioralisso, 2016, p. 3)

\subsection{Relación con el territorio donde desenvuelven sus dinámicas}

Entender la identificación de un grupo social con el territorio es necesario porque, en los procesos de constitución de identidad de sociedades locales, el componente territorial es básico. Asimismo, esa identificación se consolida si hay intercambio con otros grupos; el arraigo a un territorio se hace más si es posible la comparación, la defensa y proposición de cambios (Arocena, 2002). Así, cuando se analiza la relación de Fiteca con el territorio de La Balanza esta pasa por un doble proceso de identificación y vinculación. Por un lado, el contexto específico del lugar impacta e influye en el accionar de Fiteca, lo cual no deja de lado las dinámicas globales que moldean sus intervenciones. Al respecto Fioralisso en sus investigaciones sostiene que:

La gestión de recursos de la comunidad Fiteca está enmarcada en una tradición barrial de organización vecinal del distrito de Comas: la autogestión. La comunidad Fiteca recoge esta tradición y aplica las mismas formas organizativa y de gestión, así tenemos que recurre al trabajo voluntario de sus miembros a manera de faena e invita a participar a terceros - tanto amistades y artistas cercanos a la organización como instituciones públicas y privadas- bajo la misma modalidad[...]. (Fioralisso, 2016, p. 5)

Asimismo, Fiteca no solo se apropia e incorpora este elemento como una lógica de trabajo que va guiar sus dinámicas, sino también hay un proceso de apropiación del mismo por parte de los vecinos del barrio. Los organizadores sostienen que para los vecinos de La Balanza Fiteca ya forma parte de su tradición barrial, donde el festival es percibido como una fecha importante de celebración que está presente. En ese sentido, las dinámicas de Fiteca están influidas por la tradición barrial en cuanto a su forma de organización y los habitantes del lugar se apropiaron de ella.

Por otro lado, Fiteca también ha impactado en el territorio en la medida que se apropió de ella y lo fue transformando en el tiempo a través de diversos proyectos y actividades como el Proyecto Fitekantropus desarrollado junto al colectivo CITIO. Los organizadores mencionan que intervinieron en lugares abandonados para convertirlos en espacios de recreación para los niños, rescataron lugares poco transitables, mejoraron las calles, crearon un comedor popular, una biblioteca, etc., es decir, dotaron de color y vida al barrio, transformando sus espacios públicos para hacerlos acogedores y romper con la denominación estigmatizada de Asentamiento Humano. Asimismo, Fiteca no solo impacta transformando el espacio, sino también en el imaginario de los vecinos de La Balanza reconfigurando la identificación con su territorio a través de las prácticas culturales y artísticas que desenvuelve Fiteca y el intercambio con los diferentes actores que en ellos participan.

Al respecto, en los estudios de Fioralisso se muestra como:

Las intervenciones de Fiteca han sido el escenario de encuentros con artistas y visitantes sobre los que los vecinos han ido definiendo los términos de su relación con lo foráneo. Esta relación ha producido una respuesta en los vecinos basada en amabilidad y hospitalidad hacia los visitantes, valores que en su puesta en escena a través de los años han ido construyendo y, producto del impacto mediático en 
momentos como el festival, proyectando hacia afuera una imagen del barrio que busca contrarrestar a aquella estigmatizada en la que se le presenta violento y peligroso. (Fioralisso, 2016, p. 6)

Además, según el mismo autor, la aparición de la cultura en el barrio es atribuida a Fiteca siendo incorporada a una narrativa de progreso, donde lo cultural por momentos aparece como el punto más alto y, en otros casos, se presenta como uno de los factores que han confluido para hacer del barrio un lugar mejor. De ese modo, la identidad del territorio y el imaginario de los habitantes ha sido reconfigurado en el transcurso de los años mediante las dinámicas culturales y artísticas de Fiteca. Finalmente, todo este proceso de apropiación y transformación del territorio se ha dado con la participación activa de los vecinos de La Balanza. Este proceso también es analizado por Gutiérrez, Fontcuberta y Villar (2016), quienes sostienen lo siguiente:

La comunidad se erige en agente promotor de su propio desarrollo, social y urbano, y defensora de la ciudadanía que, gracias a la comunidad Fiteca, disfruta del arte y la cultura y es protagonista en la toma de decisiones que la afectan. (Gutiérrez, Fontcuberta y Villar, 2016, p.39)

En ese sentido, estas acciones podrían entenderse como parte de iniciativas locales, es decir, hay una movilización social que parte de iniciativas propias para transformar el territorio con el fin de mejorar sus condiciones vida, sobre todo, teniendo en cuenta que estas poblaciones forman parte del sector marginal, donde las condiciones de vida son precarias y el rol del estado es limitado.

\section{Perspectivas a futuro}

A partir de las entrevistas realizadas a distintos miembros y participantes de Fiteca, hemos podido conocer algunos planteamientos e ideas a futuro. Muchos de ellos han formado parte de este desde su infancia, en donde han podido apreciar cómo este movimiento artístico-cultural ha impactado de manera positiva en el barrio La Balanza y en los vecinos y vecinas de Comas en general.

En primer lugar, muchos de los testimonios hablan de las repercusiones positivas y la transformación que se ha visto reflejada en distintos niveles sociales donde el rol de Fiteca resultó crucial. Esto radica en la importancia y la autonomía del movimiento, cuya función gira en torno a forjar una identidad cultural que busca la contemplación de la dialéctica entre lo ancestral y lo contemporáneo. Entre los cambios en este espacio, mencionan temas como el pandillaje, el cual se vio reducido e incluso varios de los jóvenes involucrados en estos actos se incorporaron luego al grupo La Gran Marcha de los Muñecones. En segundo lugar, resalta la conversación con Richard Amancio, jefe de la comisión tramoya, respecto a cuál sería su apreciación sobre establecer una dinámica económica alternativa más estructurada dentro del festival. Propuesto eso, mencionó la prevalencia de toda una cooperación barrial para llevar a cabo la organización de la semana de Fiteca, presentándola como la base para futuras posibles dinámicas.

Por un lado, se tiene al comedor y a la comisión hospedaje para poder albergar a los artistas, además de la intervención vecinal en una feria de comida que acompaña al festival, dentro de los cuales se suscitan diversos intercambios culturales que enriquecen el conocimiento general del barrio. Esto se refleja en lo escrito por Pinedo (2015), en donde ponen en evidencia estas dinámicas de acción colectiva pues «resultan desafiantes también las lógicas de trabajo detrás de Fiteca basadas en la ofrenda que distan de las relaciones mercantiles que caracterizan las industrias culturales ... cuyo poder no solo radica en que representa un desafío a la autoridad sino en que fomenta precisamente la solidaridad» (p. 41). Es así, que dicho choque cultural entre artistas, visitantes y vecinos enriquece la comprensión de nuevas realidades y predispone a los 
vecinos y vecinas a la apreciación de nuevas formas de vida, arte y cultura. Por otro lado, se tiene la gestión interna del festival, el cual cuenta con comisiones como la de economía y la de auspiciadores que se encargan de reinventarse todos los años para poder llevarlo a cabo. Sin embargo, Richard, nos comenta que esta cuestión (económica) es difícil y que «la ideamos»» ${ }^{12}$

Fioralisso destaca la participación de Fiteca:

a través del grupo de teatro LGMM, de programas estatales de apoyo a iniciativas culturales, así como también de otros circuitos que conectan organizaciones culturales que son impulsados desde la sociedad civil. Además, a través de estas redes, es que han hecho su aparición en el barrio organizaciones no gubernamentales, organismos de financiamiento internacionales y empresas privadas que, con el discurso de la cultura para el desarrollo y la responsabilidad social empresarial, han buscado ejecutar proyectos de desarrollo en la localidad. $(2016$, p.5)

Esto refleja que existen auspiciadores - Anypsa, PUCP, Walok, entre otros - y alguna suerte de ayuda por parte de la Municipalidad Distrital de Comas y el Ministerio de Cultura, sin embargo, el festival ha alcanzado tal importancia que se necesita ir más allá, sin perder la característica de la autogestión y la acción colectiva.

De manera más concisa, tenemos a Fiteca dentro de un espacio que se ha visto influenciado de manera positiva ante la cultura y la transformación de la autopercepción del barrio. Sin embargo, siguen existiendo variables que limitan el crecimiento de esta iniciativa cultural. Por un lado, ellos buscan que este movimiento trascienda más allá de los límites de Comas, pues si bien ya tienen presencia en otros barrios, sienten la responsabilidad de llegar a más gente que no tiene acceso directo al arte y a la cultura en cualquiera de sus facetas. Esto mediante la movilización intermitente de Fiteca con un carácter sostenible que brinde las condiciones para que la comprensión - de los distintos barrios - de las intencionalidades que tienen como movimiento cultural, sea orgánica.

Por otro lado, Jorge Rodríguez tiene claro que «se debe tocar la economía» ${ }^{13}$ y esto se sugiere mediante la estructuración de un plan económico que vaya de acuerdo a sus ideales y como movimiento logren una evolución digna. A su vez, Rodríguez también propone que la responsabilidad sea tomada por estudiantes universitarios «que tengan una perspectiva fresca y que tengan la vocación, para influir en la desconfianza que existe respecto a estos temas» ${ }^{14}$. Y aunque se haya hecho mención de las dificultades y obstáculos para llevarlo a la práctica, cada vez es más necesario repensar soluciones para que el siguiente paso que debe tomar Fiteca sea el ideal para todo el movimiento. Es cuestión de mirar a la cultura como condición para la dignidad y la vida.

\section{Conclusiones}

Para cerrar esta investigación, es importante mencionar a manera de respuesta a nuestras interrogativas y de manera concluyente, lo siguiente:

Fiteca desenvuelve prácticas artístico-culturales basadas en lógicas de cooperación y el trabajo voluntario de sus miembros, vecinos/as y artistas como pilares fundamentales de

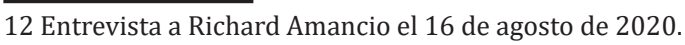

13 Entrevista a Jorge Rodríguez el 27 de junio de 2020.

14 Entrevista a Jorge Rodríguez el 27 de junio de 2020. 
producción y reproducción de sus dinámicas que, con el tiempo, han reconfigurado la identidad territorial y la imaginación de sus habitantes; dado que, estas relaciones solidarias ha fortalecido los vínculos entre los actores y ha transformado la intervención de Fiteca mediante un proceso de apropiación recíproca entre Fiteca y el barrio, dando como resultado la construcción de la comunidad Fiteca. Una comunidad constituida por diversos actores, tanto artistas como vecinos/ as del barrio, que se sostiene en vínculos de cooperación y el trabajo voluntario.

Así, estas relaciones colectivas y de trabajo voluntario pondera lógicas contrarias a la lógica utilitarista y de individuación subyacente al sistema capitalista y la economía de mercado, con lo cual se vincula más a las lógicas de una economía social, pero cobra mayor fuerza si tomamos en cuenta que en esta propuesta la economía, la sociedad y la cultura están estrechamente vinculados, es decir, las prácticas económicas no pueden ser ajenas a los demás ámbitos, ya que, están estrechamente relacionadas.

En ese sentido, se puede inferir que las relaciones económicas sociales que desenvuelve Fiteca no solo impacta en la dimensión física del barrio de La Balanza, sino también en lo simbólico reconfigurando la identidad territorial y la imaginación de sus habitantes.

Asimismo, la organización interna de la comunidad Fiteca tiene un basamento democrático en la medida que recoge las opiniones e inquietudes de todos los miembros y participantes, a través de asambleas generales, además de informar de los avances y detalles de los proyectos, donde cada uno y cada una tiene derecho a voz y voto para construir los sueños que los une, tal como refieren sus organizadores. Asimismo, para el desarrollo de las actividades se organizan grupos de trabajo, donde cada uno se integra en función a la afinidad con determinada área.

Esta forma de organización comparte características con el gobierno interno de la economía social descrita por Coraggio (2011), pues esta lógica de cooperación, es una práctica social, porque genera valores de uso para satisfacer las necesidades de sus comunidades y no está orientada por la ganancia; y los agentes económicos, no son escindidos de sus identidades sociales, de su historia ni de su incrustación en el mundo simbólico e institucional que denominamos cultura, por lo que, es entendida como un espacio de acción constituido por individuos, familias, comunidades y colectivos que se mueven dentro de instituciones decantadas por la práctica o acordadas como arreglos voluntarios, que actúan haciendo transacciones entre la utilidad material y los valores de solidaridad y cooperación, limitando la competencia.

Asimismo, el gobierno interno de sus prácticas se basa en la deliberación entre miembros que tienen cada uno un voto; pero admite la división del trabajo y los sistemas de representación y control de las responsabilidades, de ese modo, la democracia participativa es su recurso fundamental para que no se plantee apolítico, sino pro democracia participativa. (Coraggio, 2011)

De otro lado, en función a los valores que persigue Fiteca, de acuerdo a lo señalado por los organizadores y la puesta en práctica a través de diversos proyectos, y la formas de organización con participación activa de los vecinos de La Balanza, como agentes de cambio y transformación de su propio espacio físico y social, se podría hablar de un modo de desarrollo alternativo; ya que, según la descripción de Santos (2011), este es concebido como una forma de promover mejores condiciones de vida para la población en general, y para los sectores marginados en particular, donde lo económico es parte integral y dependiente de lo social y subordinado a la protección de bienes y valores no económicos como lo cultural. Además, esta perspectiva plantea un desarrollo de base o de abajo hacia arriba, es decir, desde la sociedad civil. 
También, como hemos anotado desde el inicio de esta investigación, una parte fundamental para lograr ese nivel de organización y organicidad dentro de la comunidad ha sido la deconstrucción generada por la apertura crítica que brinda el teatro popular, por sus características de contenido y objetivos que persigue, impulsado por el colectivo La Gran Marcha de los Muñecones, en este caso particular, es esta revalorización lo que ha impulsado la creación de esta identidad llamada Fiteca, para los vecinos y las vecinas de La Balanza.

En ese sentido, las prácticas artístico-culturales y las dinámicas desenvueltas en torno a ellas por la comunidad Fiteca, en el barrio de La Balanza, comparten lógicas con las formas económicas alternativas como la economía social en cuanto a la organización del trabajo, la coordinación, toma de decisiones y los principios que guían sus intervenciones, así como partir de iniciativas sociales de base y la configuración de la identidad territorial. Sin embargo, estas prácticas no están al margen de las dinámicas de mercado, sino que coexisten con ellas, ya que, en cierta medida estas prácticas se combinan.

\section{Referencias}

Arocena, J. (2002). El desarrollo local: un desafío contemporáneo. Caracas: Nueva Sociedad.

Benza, R. y Rosas, C. (2011). Temas y personajes marginales en el teatro peruano contemporáneo. En Claudia Rosas, La marginación en el Perú. Siglo XVI a XXI (pp. 405-432). Lima: Pontificia Universidad Católica del Perú.

Bild, A. E. (2012). Por el Amor del Arte: Kā teātris ietekmē kopienu. Teātra pārveidotas apkaimes etnografía (tesis de maestría). Universidad de Letonia, Riga.

Casallo, V. (2013). Estética y espacio público: una lectura de Hannah Arendt desde la comunicación. Conexión, (2), 98-111.

Cunyas Mendoza, L. S. y Cárdenas Olivera, S. J. (2018). Rehabilitación de espacios públicos y centro de artes especializado en teatro en el barrio La Balanza-Comas. https://bit.ly/2ZQZVHK

Coraggio, J. L. (2011). La Economía Social como vía para otro desarrollo social. En A. Acosta y E. Martínez (Eds.). Economía Social y Solidaria. El trabajo antes que el capital. Quito: Ediciones Abya- Yala.

Cubas, J. V. Regeneración urbana, espacios públicos y cultura viva. En Ciudades Vivibles (pp. 21-24). Guadalajara: Forópolis.

Fioralisso Abraham, P. (2016). Sembrando barrios culturales: arte y comunidad en el barrio de La Balanza en Comas (tesis de licenciatura). Pontificia Universidad Católica del Perú, Lima.

Gabella, E. C. (2017, December). La construcción participativa del espacio público en la ciudad informal. El caso del local comunal del barrio de la balanza (distrito de Comas, Lima). In Actas Congreso Iberoamericano Redfundamentos (No. 1, pp. 819-831).

Germaná, C (2015). La economía de la reciprocidad y el Buen Vivir. En H. Cottyn, J. Jahancke, L. Montoya, E. Pérez y M. Tempelmann (Comps.), Las luchas sociales por la tierra en América Latina. Un análisis histórico, comparativo y global (pp. 173-182). Lima: Fondo de Investigación de Flandes, Facultad de Ciencias Sociales de la Universidad Mayor de San Marcos, Red Muqui.

Gonzales, N., Gonzales, J., Guerra, G. y Valle, S. (2012). La Gran Marcha de Muñecones: una experiencia estética. Facultad de ciencia y artes de la comunicación. Lima: Pontificia Universidad Católica del Perú.

Gutiérrez-Mozo, M. E.; Fontcuberta Rubio, D. y Villar Pastor, P. (2016). Lima participada y participativa: el renacer del Barrio Cultural de La Balanza, Distrito de Comas. Lima. (pp. 109-123). En Jaume Blancafort y Patricia Reus (Eds. y coords.), La participación en la construcción de la ciudad. Cartagena: Universidad Politécnica de Cartagena.

Malca Vargas, M. M. (2008). La gente dice que somos teatro popular: referentes de identidad en la práctica teatral de la zona periférica de Lima Metropolitana (tesis de licenciatura). Pontificia Universidad Católica del Perú, Lima. 
Merladet, S. (2017). “El método del elefante”. Análisis de los procesos de participación en proyectos arquitectónicos de mejora, La Balanza, Lima. Madrid: Escuela Técnica Superior de Arquitectura de Madrid (ETSAM).

Navarro García, G. (2014). Escuela de artes escénicas en Lima norte: el espacio abierto como escenario. https:// bit.ly/3EEc6Xf

Pinedo, N. E. (2015). "Hacer de la calle un verso, y del barrio, poesía”: dinámicas de cooperación a partir de la institucionalización de FITECA en el barrio La Balanza, Comas. La Colmena, (8), 28-41.

Quijano, A. (2014). Cuestiones y Horizontes. De la Dependencia Histórico-Estructural a la Colonialidad/ Descolonialidad del Poder. Buenos Aires: CLACSO.

Quijano, A. (2011). ¿Sistemas alternativos de producción? En B. S. Santos (coord.). Producir para vivir. Los caminos de la producción no capitalista (pp. 369-399). México D.F.: Fondo de Cultura Económica.

Rodríguez, F. A. A. (2020). Infraestructuras evolutivas arquitectura para el desarrollo en los barrios a partir de la cultura popular (tesis doctoral). Pontificia Universidad Católica del Perú, Lima.

Rodríguez Bernu, F. (2015, 20 de octubre). Arquitectura en comunidad: FITECA y la creación de los Barrios Culturales de La Balanza en Lima 19 oct 2015. ArchDaily Perú. https://bit.ly/3bxbqGR

Ruiz Fernández, D. R., y Benites Arrieta, M. P. (2020). Capital social y desarrollo local. El caso del Proyecto Fitekantopus en el Barrio Cultural de La Balanza-Comas (tesis de maestría). Pontificia Universidad Católica del Perú, Lima.

Santos, B. S.; Rodríguez, C. (2011). Introducción. Para ampliar el canon de la producción (pp. 15-62). En B. S. Santos (coord.). En Boaventura de Sousa (Coord.). Producir para vivir. Los caminos de la producción no capitalista. México D.F.: Fondo de Cultura Económica.

Torres del Castillo, S. (2018). Centro de formación y difusión de las artes escénicas en el barrio de La Balanza, Comas. Lima. https://bit.ly/31pJRNQ

Villarán, S.; Alayza, P. P.; Méndez, G. M. L.;Valdizan, G. (2012). I Seminario Internacional de Cultura Viva Comunitaria. https://bit.ly/31pK7wi

Presentado: 12/05/2021

Aceptado: 05/06/2021

Publicado online: $27 / 12 / 2021$ 